\title{
Differences between myeloperoxidase-antineutrophil cytoplasmic autoantibody (ANCA) and proteinase 3-ANCA associated vasculitis: A retrospective study from a single center in China
}

\author{
TING WU ${ }^{1}$, CHANJUAN SHEN ${ }^{2}$, YONG ZHONG ${ }^{1}$, JOSHUA D. OOI ${ }^{3}$, YA-OU ZHOU ${ }^{4}$, JIN-BIAO CHEN ${ }^{5}$, \\ TING MENG $^{1}$, ZHOU XIAO ${ }^{1}$, WEI LIN ${ }^{6}$, XIANG AO ${ }^{1}$, XIANGCHENG XIAO ${ }^{1}$, \\ QIAOLING ZHOU ${ }^{1}$ and PING XIAO ${ }^{1}$

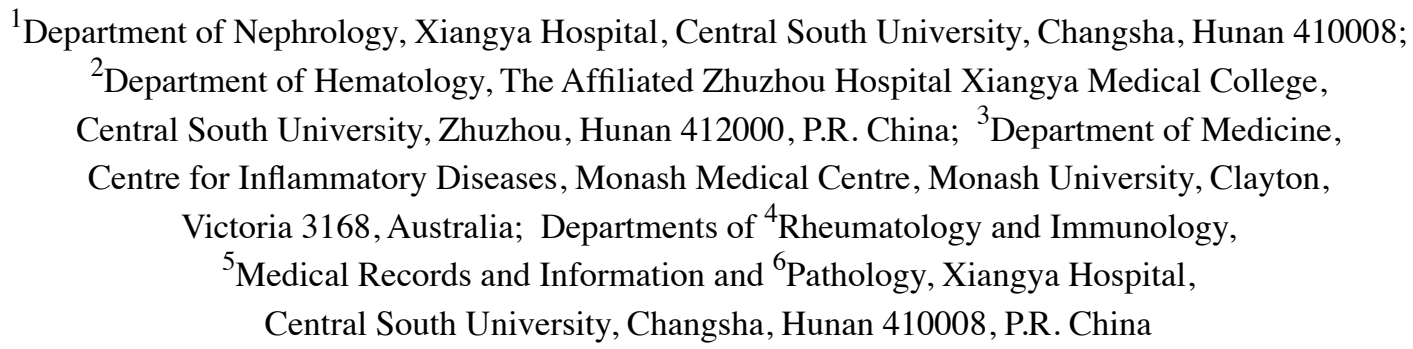

Received March 2, 2020; Accepted January 12, 2021

DOI: $10.3892 /$ etm.2021.9993

\begin{abstract}
In antineutrophil cytoplasmic autoantibody (ANCA)-associated vasculitides (AAV), the two major target antigens of ANCA are proteinase 3 (PR3) and myeloperoxidase (MPO). Evidence is accumulating that there are distinct differences between patients with PR3-AAV and those with MPO-AAV. In the present study, the clinicopathological features and prognosis of patients with PR3-AAV and MPO-AAV from a single center in China were retrospectively analyzed. A total of 212 Chinese patients with AAV were recruited in the present study; 189/212 (89.15\%) patients were classified as having MPO-AAV and 23/212 (10.85\%) patients as having PR3-AAV. Compared with those in the PR3-AAV group, patients in the MPO-AAV group were older and less frequently had ear, nose and throat or ophthalmic involvement. MPO-AAV patients had higher levels of serum creatinine and proteinuria at baseline. No significant difference was observed with regard to the pathological changes of the glomeruli and tubulointerstitium between the two groups. The probability of developing end-stage renal disease was significantly higher in patients with MPO-AAV compared with that in patients with PR3-AAV. There was no significant difference in the one-year
\end{abstract}

Correspondence to: Dr Yong Zhong or Professor Ping Xiao, Department of Nephrology, Xiangya Hospital, Central South University, 87 Xiangya Road, Changsha, Hunan 410008, P.R. China E-mail: zhongyong121@163.com

E-mail: xiaoping.x@163.com

Key words: antineutrophil cytoplasmic autoantibody, myeloperoxidase, proteinase 3 , clinicopathological features, prognosis patient survival rate between the two groups. However, differences in certain clinical characteristics and outcomes were observed between MPO-AAV and PR3-AAV patients. A large national investigation of AAV is required to confirm the concept that PR3-AAV and MPO-AAV are distinct disease entities.

\section{Introduction}

Antineutrophil cytoplasmic autoantibody (ANCA)-associated vasculitides (AAV) are characterized by necrotizing inflammation of small-to-medium-sized vessels, resulting in a distinctly heterogeneous clinical manifestation (1). The first formal criteria for the classification of vasculitis were published in 1990 by the American College of Rheumatology and did not include ANCA (2). According to the 2012 Chapel Hill Consensus Conference, AAV has been classified into three major types: Granulomatosis with polyangiitis (GPA), microscopic polyangiitis (MPA) and eosinophilic GPA (3). A recent genome-wide association study of AAV has suggested that MPO-AAV and PR3-AAV have distinct genetic causes, which supports the concept that MPO-AAV and PR3-AAV are distinct autoimmune syndromes (4).

Previous studies have indicated that the epidemiology, genetics, clinical manifestations and prognosis are different between MPO-AAV and PR3-AAV in European countries and that patients with PR3-AAV have a higher likelihood of relapse than those with MPO-AAV (5). Fussner et al (6) reported that a higher PR3-ANCA titer during complete remission was associated with an increased risk of relapse and that MPO-AAV was associated with poor prognosis regarding patient survival. However, a recent study from France suggested that the clinicopathological classification appears to be superior to antibody specificity in predicting prognosis in patients with AAV (7). 
A recent retrospective monocentric study suggested that the epidemiological characteristics, clinical histopathological features and outcomes were different between MPO- and PR3-positive Chinese patients with GPA (8). Therefore, the purpose of the present study was to investigate whether the clinical and pathological characteristics/outcomes were different between patients with MPO-AAV and PR3-AAV in our center.

\section{Materials and methods}

Patients and data collection. A total of 212 consecutive patients were diagnosed with AAV at Xiangya Hospital of Central South University (Changsha, China) between January 2008 and January 2018. All patients fulfilled the Chapel Hill Consensus Conference criteria for AAV (3) and were identified as ANCA-positive by ELISA during the course of their disease. According to ANCA specificity, AAV was divided into MPO-AAV and PR3-AAV. The patients were enrolled in either the MPO-AAV group or the PR3-AAV group. The exclusion criteria were as follows: i) Patients with coexistence of other multisystem autoimmune diseases or kidney diseases; ii) hepatitis $\mathrm{B}$ or $\mathrm{C}$ virus or HIV infection; iii) double-positive for MPO-ANCA and PR3-ANCA or anti-Glomerular basement membrane (GBM) positive. Baseline demographics, clinical and laboratory data and treatment information were obtained from the electronic medical record system of the hospital. The Birmingham Vasculitis Activity Score (BVAS) was used to assess AAV activity (9).

Renal histopathology. Renal specimens of 88 of the 212 patients with AAV were evaluated by direct immunofluorescence and light and electron microscopy (10). The biopsy specimens were assigned into 4 categories, namely focal, crescentic, mixed and sclerotic, according to the definition of the 2010 histological classification (11). Interstitial and tubular lesions were scored semi-quantitatively, as described previously (12). Biopsies were independently scored by two pathologists who were blinded to the clinical data.

Treatment. The standard induction therapy consisted of oral prednisone plus cyclophosphamide (CTX) $(13,14)$. Patients received prednisolone at the initial dose of $1 \mathrm{mg} / \mathrm{kg} /$ day orally for 2 weeks, and prednisolone was tapered to $10-12.5 \mathrm{mg}$ per patient per day by 3 months. CTX was administered intravenously $0.5-0.75 \mathrm{~g} / \mathrm{m}^{2}$, once every month. Dosages of CTX were adjusted to maintain the leukocyte count $>4 \times 10^{9} / 1$. For patients with AAV older than 65 years or those with a glomerular filtration rate (GFR) $<20 \mathrm{ml} / \mathrm{min}$ per $1.73 \mathrm{~m}^{2}$, a $25 \%$ dose reduction of CTX was performed. Methylprednisolone pulse therapy and/or additional plasma exchanges were given to certain patients with rapidly progressive glomerulonephritis or pulmonary hemorrhage prior to the standard induction therapy. Intravenous CTX every 3 months or daily oral azathioprine or mycophenolate mofetil was given for maintenance therapy.

Follow-up. The patients were followed up until death, progression to end-stage renal disease (ESRD), or the final follow-up date (January 30, 2019). ESRD was defined as estimated GFR $<15 \mathrm{ml} /\left(\min \times 1.73 \mathrm{~m}^{2}\right)$ or maintenance of renal replacement therapy for $>3$ months. All follow-up data were collected from the hospital's electronic medical records and by contacting the individual patients directly.

Statistical analysis. All data were analyzed using the statistical software SPSS, version 23.0 (IBM Corp.). Categorical data are expressed as $\mathrm{n}(\%)$ and the results were compared using the $\chi^{2}$ test or Fisher's test. Quantitative data are expressed as the mean \pm standard deviation (for normally distributed data) or median with interquartile range (for non-normally distributed data) and the results were compared by Student's t-test or the Mann-Whitney U test, respectively. Kaplan-Meier curves and log-rank tests were used to analyze patient survival and renal survival. Patients who were lost to follow-up before the final follow-up date of this study (January 30, 2019) were censored. Those who survived or didn't progress to ESRD at end of study were also censored from the survival analysis. $\mathrm{P}<0.05$ was considered to indicate statistical significance.

\section{Results}

Patient baseline demographic. As presented in Table I, of the 212 patients with AAV diagnosed between January 2008 and January 2018 at Xiangya Hospital of Central South University (Changsha, China), 189 (89.15\%) patients were classified as having MPO-AAV and $23(10.85 \%)$ as PR3-AAV. Among the 189 patients who were MPO-ANCA-positive, 4 (2.10\%) patients were diagnosed with GPA. Among the 23 patients who were PR3-ANCA positive, 11 (47.80\%) patients were diagnosed with MPA. The incidence of MPA was higher in the MPO-AAV group compared with that in the PR3-AAV group (97.90\% vs. $47.80 \% ; \mathrm{P}<0.001)$. No significant difference in the sex distribution was present between the MPO-AAV (102/87) and PR3-AAV (13/10) groups. Compared with the patients in the PR3-AAV group, patients in the MPO-AAV group were older $(\mathrm{P}<0.01)$.

Patient clinical and laboratory data. Patients in the MPO-AAV group had more severe renal damage compared with those in the PR3-AAV group. The average serum creatinine level in the MPO-AAV group was higher than that in the PR3-AAV group $(406 \pm 288$ vs. $170 \pm 134 \mu \mathrm{mol} / 1 ; \mathrm{P}<0.01)$. Compared with those in the MPO-AAV group, the PR3-AAV group had significantly higher levels of serum complement $3(\mathrm{C} 3 ; \mathrm{P}=0.022)$ and C-reactive protein (CRP; $\mathrm{P}<0.01)$ and lower 24 -h proteinuria $(\mathrm{P}=0.023)$. There were no differences in the median BVAS, serum $\mathrm{C} 4$, hemoglobin or serum albumin levels between the groups. The most common organ involved in MPO-AAV and PR3-AAV was the kidney and no significant difference was present between the groups $(\mathrm{P}=0.143)$. There was no difference regarding lung involvement between the groups. The incidence of ENT involvement (11.64 vs. 43.48\%, $\mathrm{P}<0.01)$ and ophthalmic involvement (6.35 vs. $43.48 \%, \mathrm{P}<0.01)$ was significantly lower in the MPO-AAV group compared with that in the PR3-AAV group.

Renal histopathology. Renal biopsies were performed in 80 patients with MPO-AAV and 8 patients with PR3-AAV. 
Table I. Baseline demographic and clinical parameters of patients with AAV.

\begin{tabular}{|c|c|c|c|c|}
\hline Parameter & Total $(n=212)$ & MPO-AAV (n=189) & PR3-AAV (n=23) & P-value \\
\hline Age (years) & $58.05 \pm 15.02$ & $59.61 \pm 13.83$ & $45.13 \pm 18.22$ & $<0.001$ \\
\hline Males/females & $115 / 97$ & $102 / 87$ & $13 / 10$ & 0.816 \\
\hline Duration of disease (days) & $64(37,150)$ & $70(36,150)$ & $60(37,90)$ & 0.583 \\
\hline MPA & $196(92.45)$ & $185(97.90)$ & $11(47.80)$ & $<0.001$ \\
\hline \multicolumn{5}{|l|}{ Organ involvement } \\
\hline Pulmonary & $139(65.57)$ & $122(64.55)$ & $17(73.91)$ & 0.372 \\
\hline ENT & $32(15.09)$ & $22(11.64)$ & $10(43.48)$ & $<0.001$ \\
\hline Ophthalmic & $22(10.38)$ & $12(6.35)$ & $10(43.48)$ & $<0.001$ \\
\hline Gastrointestinal & $18(8.49)$ & $15(7.94)$ & $3(13.04)$ & 0.407 \\
\hline Nervous system & $48(22.64)$ & $43(22.75)$ & $5(21.74)$ & 0.913 \\
\hline Renal & $199(93.87)$ & $179(94.71)$ & $20(86.96)$ & 0.143 \\
\hline BVAS & $13.37 \pm 5.54$ & $13.18 \pm 5.70$ & $14.86 \pm 3.65$ & 0.060 \\
\hline Serum creatinine $(\mu \mathrm{mol} / \mathrm{l})$ & $380 \pm 250$ & $406 \pm 288$ & $170 \pm 134$ & $<0.001$ \\
\hline Urinary protein $(\mathrm{g} / 24 \mathrm{~h})$ & $0.74(0.21,1.5)$ & $0.84(0.25,1.63)$ & $0.23(0,1)$ & 0.023 \\
\hline Serum albumin $(\mathrm{g} / \mathrm{l})$ & $29.49 \pm 6.25$ & $29.47 \pm 6.18$ & $29.60 \pm 6.94$ & 0.884 \\
\hline Hemoglobin & $82.69 \pm 21.24$ & $81.57 \pm 20.79$ & $91.87 \pm 23.11$ & 0.053 \\
\hline Serum C3 (g/l) & $832 \pm 286$ & $810 \pm 251$ & $1021 \pm 464$ & 0.022 \\
\hline Serum C4 (g/l) & $238 \pm 100$ & $237 \pm 95$ & $242 \pm 139$ & 0.563 \\
\hline $\mathrm{CRP}(\mathrm{mg} / \mathrm{l})$ & $26(7.2,81.0)$ & $22.5(6.7,76.0)$ & $67(24.0134 .0)$ & 0.002 \\
\hline
\end{tabular}

Values are expressed as the mean \pm standard deviation, the median (interquartile range) or $\mathrm{n}(\%)$. AAV, antineutrophil cytoplasmic autoantibody-associated vasculitides; PR3, proteinase 3; MPO, myeloperoxidase; MPA, microscopic polyangiitis; BVAS, Birmingham Vasculitis Activity Score; ENT, ear, nose and throat; C3, complement 3; CRP, C-reactive protein.

Table II. Renal pathological features of patients with AAV.

\begin{tabular}{lccr}
\hline Item & MPO-AAV $(\mathrm{n}=80)$ & PR3-AAV $(\mathrm{n}=8)$ & P-value \\
\hline Sclerotic class & $17(21.25)$ & $0(0.00)$ & 0.326 \\
Focal class & $12(15.00)$ & $2(25.00)$ & 0.818 \\
Crescentic class & $28(35.00)$ & $4(50.00)$ & 0.649 \\
Mixed class & $23(28.75)$ & $2(25.00)$ & 1.000 \\
Tubule-interstitial lesion score & $1(1,2)$ & $1.5(1,2)$ & 0.813 \\
\hline
\end{tabular}

Values are expressed as $\mathrm{n}(\%)$ or the median (interquartile range). AAV, antineutrophil cytoplasmic autoantibody-associated vasculitides; PR3, proteinase 3; MPO, myeloperoxidase.

Although there was no significant difference in renal histopathology between the groups, there was a trend of the proportion of sclerotic class cases in the MPO-AAV group (21.25\%) being higher than that in the PR3-AAV group (0.00\%) and the proportion of crescentic class cases in the PR3-AAV group $(50.00 \%)$ being higher than that in the MPO-AAV group (35.00\%; Table II). Renal histopathology images from a case of MPO-AAV and a case of PR3-AAV assessed using immunofluorescence and light and electron microscopy images are presented in Fig. S1.

Patient and renal survival. As presented in Table III, $174(92.06 \%)$ patients with MPO-AAV and $22(95.65 \%)$ patients with PR3-AAV received immunosuppressive treatment, which consisted of steroids plus cyclophosphamide in $111(63.79 \%)$ patients with MPO-AAV and $15(65.22 \%)$ patients with PR3-AAV, steroids plus other immunosuppressant (rituximab or mycophenolate) in $13(7.47 \%)$ patients with MPO-AAV and $2(8.70 \%)$ patients with PR3-AAV and steroids alone in $50(28.74 \%)$ patients with MPO-AAV patients and 5 (22.73\%) patients with PR3-AAV. In total, 49 patients received plasma exchange. Among the 212 patients with AAV, 36 patients with MPO-AAV and 5 patients with PR3-AAV were lost to follow-up. In total, $44.3 \%$ of the patients in the MPO-AAV group and $26.7 \%$ of the patients in the PR3-AAV group progressed to ESRD in the first year $(\mathrm{P}<0.001)$. As presented in Fig. 1, the 1-year kidney survival rate was significantly higher in the PR3 group than that in the MPO group. No 
Table III. Treatment of patients with AAV.

\begin{tabular}{|c|c|c|c|}
\hline Treatment & MPO-AAV (n=189) & PR3-AAV $(n=23)$ & P-value \\
\hline Immunosuppressive treatment & $174(92.06)$ & $22(95.65)$ & 0.538 \\
\hline Steroids plus cyclophosphamide & $111(58.73)$ & $15(65.22)$ & 0.686 \\
\hline Steroids plus other immunosuppressant & $13(6.88)$ & $2(8.70)$ & 0.788 \\
\hline Steroids alone & $50(26.46)$ & $5(21.73)$ & 0.555 \\
\hline Plasma exchange & $45(23.81)$ & $4(17.39)$ & 0.491 \\
\hline
\end{tabular}

Values are expressed as $\mathrm{n}(\%)$ or the median (interquartile range). AAV, antineutrophil cytoplasmic autoantibody-associated vasculitides; PR3, proteinase 3; MPO, myeloperoxidase.

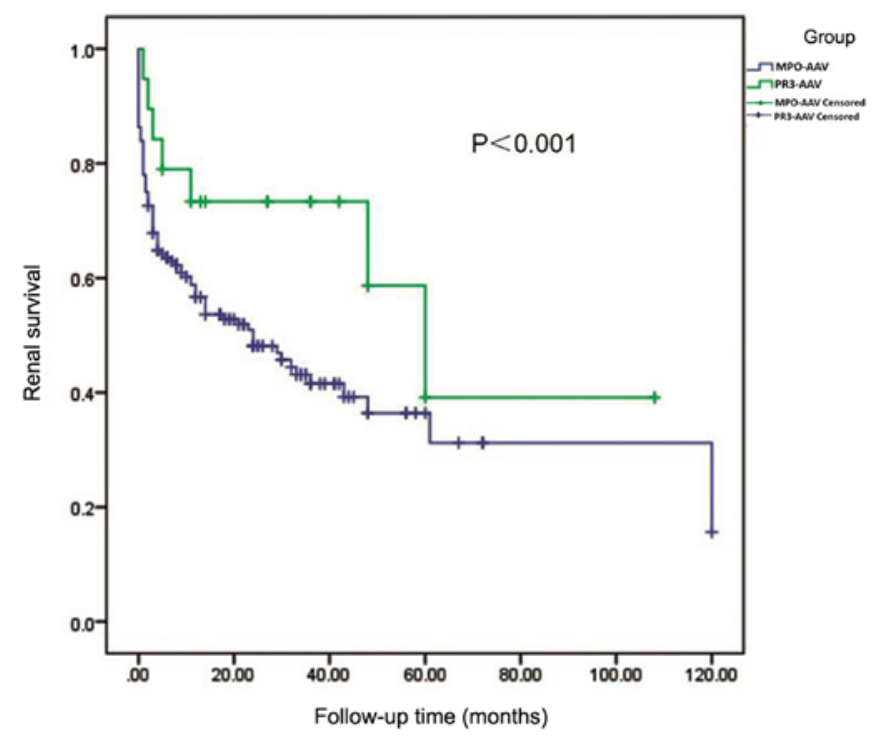

Figure 1. Renal survival of patients with different types of AAV. Follow-up time (months) refers to the time since the start of treatment. AAV, antineutrophil cytoplasmic autoantibody-associated vasculitides; PR3, proteinase 3; MPO, myeloperoxidase.

significant difference in patient survival was present between the groups (Fig. 2).

\section{Discussion}

The prevalence and incidence of PR3-AAV and MPO-AAV are different in different countries (15-17). PR3-AAV is more common in northern Europe (18). Several studies have highlighted that MPO-AAV is dominant and more commonly present in China $(8,19,20)$, which is consistent with the results of the present study. In the present cohort of patients with AAV, $89.15 \%$ were MPO-ANCA positive. This difference between countries may be attributable to genetic pools and the environment $(4,21)$. The elderly have an increased susceptibility to AAV, without any differences in ANCA specificity; certain studies have reported no age differences between PR3-AAV and MPO-AAV patients $(5,22)$. However, in the present study patients with MPO-AAV were older compared with those with PR3-AAV, which was consistent with the results of another study (8). The possible reasons for this discrepancy may be due to population differences (23).

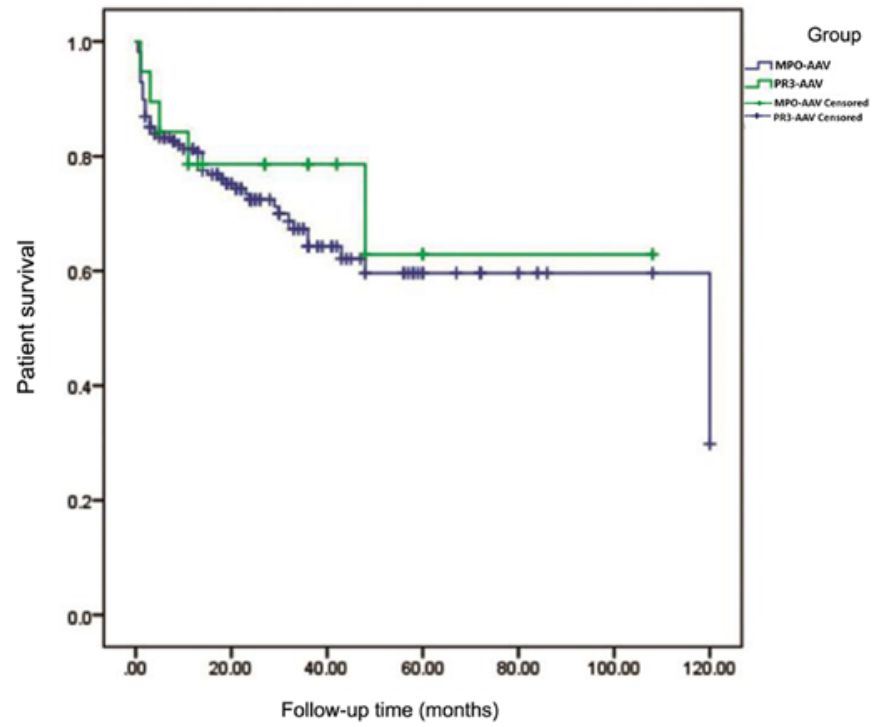

Figure 2. Patient survival of cases with different types of AAV. Follow-up time (months) refers to the time since the start of treatment. AAV, antineutrophil cytoplasmic autoantibody-associated vasculitides; PR3, proteinase 3; MPO, myeloperoxidase.

The diagnosis of AAV is based on clinical features and the presence of ANCA. AAV is a systemic disease and due to this feature, its diagnosis and determination of classification may be delayed. However, certain manifestations, such as orbital granuloma, may also occur and are suggestive of GPA (24). The clinical characteristics of AAV correlate with ANCA specificity: PR3-AAV is associated with respiratory tract involvement and more extrarenal organ manifestation $(22,25)$, while MPO-AAV is commonly a renal-limited disease $(7,26)$. The incidence of pulmonary involvement is similar in the two serotypes of AAV, but cavitary lesions and nodules are common in PR3-AAV and pulmonary infiltrates are frequently observed in MPO-AAV (27). No significant difference in pulmonary involvement was observed among the subsets in the present study.

Over $80 \%$ of patients with AAV have kidney involvement. The prevalence of renal involvement in patients with MPO-AAV was slightly higher compared with that in patients with PR3-AAV in the present study, though the difference did not reach statistical significance, which was in agreement with previous studies $(8,25,28)$. The use of ANCA to monitor 
disease activity and predict relapses in patients with renal involvement is helpful $(29,30)$. The ANCA serotype was a better predictor of relapse in remission patients than disease classification (30). MPO-ANCA is more common in MPA, while PR3-ANCA is more common in GPA. Serum ANCA is considered to be a basis for the clinical and histological classification of AAV. In European patients, 40-80\% of MPA cases were MPO-ANCA-positive and $80-95 \%$ of GPA cases were PR3-ANCA-positive (24). However, in China, MPO-ANCA positivity was estimated to be present in $>60 \%$ of GPA cases and $68.4 \%$ of the patients in the PR3 group had MPA (8). In the present study, $>90 \%$ of MPO-positive patients were diagnosed with MPA and $47.8 \%$ of PR3-ANCA-positive patients had MPA, which was in line with the data of the above-mentioned Chinese study (8). It may be speculated that such differences were due to the following reasons. First, the inclusion of only small numbers of PR3-ANCA-positive patients in the Chinese study resulted in an inaccurate representation of the group. Furthermore, ethnic differences may also have a crucial role.

Whether renal disease is tubulointerstitial or glomerular, complement has an essential role $(31,32)$. There has been an increasing interest in the role of complement in AAV in recent years. Evidence from clinical observations and animal models suggests that the alternative complement pathway has a crucial role in the pathogenesis of AAV $(10,33)$. Low serum C3 levels at diagnosis have been reported to be correlated with poor renal outcome $(34,35)$. Furthermore, patients who were MPO-ANCA-positive had lower serum C3 and C4 levels compared with PR3-ANCA-positive patients, which suggests that patients with MPO-AAV may have a more severe disease state or the presence of differences in the activated alternative pathway between ANCA serotypes. As CRP is a widely used noninvasive marker of inflammation and BVAS is the most commonly used index to evaluate the disease activity of AAV, the present data indicating that BVAS and CRP were higher in patients with PR3-AAV compared with those in patients with MPO-AAV imply that patients with PR3-AVV at diagnosis were in a more acute state of injury, while MPO-AVV patients were in a more chronic state.

Renal biopsy taken in a clinical setting may offer patients potential benefits (36). The histopathological classification of AAV proposed by Berden et al (11) in 2010 was based on the percentage of glomeruli involved. The important role of the renal pathology in AAV is to assist in diagnosis and to predict renal outcome. One study reported that crescentic class and acute tubulointerstitial lesions are more common in PR3-AAV glomerulonephritis (8). Another study revealed that although there were no differences in sclerotic, focal, crescentic or mixed classes between the groups, PR3-AAV featured a higher proportion of normal glomeruli compared with MPO-AAV (7). By contrast, fibrotic changes were more common in MPO-AAV (7). Renal histopathological classification and tubulointerstitial lesions in PR3-AAV and MPO-AAV are not found to be different at our center in the present study. The discrepancy between the present study and previous studies may be explained by an enhancement in the understanding of MPO-AAV and the utility of ANCA in making an early diagnosis of MPO-AAV (37). The number of patients with PR3-AAV with renal biopsy is small in the present study, which may result in inaccurate results. A larger number of patients with PR3-AAV with renal biopsy require to be recruited in future studies to come to a precise conclusion. The use of effective immunosuppressive treatments has improved the outcome of AAV. There are still certain disputes regarding the association between the ANCA serotype and renal and patient prognosis. Certain studies suggested that no difference in renal survival was present between ANCA serotypes $(8,28,38,39)$. However, in agreement with the results of certain previous studies $(40,41)$, the present data demonstrated that renal survival was unfavorable in patients with MPO-AAV compared with that in patients with PR3-AAV, possibly as the result of worse renal function in patients with MPO-AAV at the time of diagnosis. Another plausible explanation for this difference may be the increased proteinuria in MPO-AAV, as proteinuria has been suggested to be an independent risk factor for renal survival (42). Previous studies have suggested that there was no significant difference in patient survival between MPO-AAV and PR3-AAV patient groups $(41,43)$. The present study also indicated no association between ANCA specificity and patient survival, but 1 -year patient survival was lower than that in other centers $(7,40)$. A higher proportion of renal involvement and the lower proportion of patients who received plasma exchange may be important reasons for these observations.

There are certain limitations to this study. First, because PR3-AAV has a low prevalence in China compared to that in other countries, the number of patients in the PR3-AAV group of the present study was small. Furthermore, due to the retrospective nature of data collection, the maintenance of the immunosuppressive regimen in the two groups was not consistent. Finally, follow-up was limited in the present study.

In conclusion, MPO-AVV remains the most common type of AAV in China. Certain characteristic clinical and outcome differences may be observed and classification of patients based on the ANCA serotype appears to bring additional value to clinical practice, but it remains difficult to distinguish between MPO-AAV and PR3-AAV. A large national investigation of AAV is required to prove the concept that PR3-AAV and MPO-AAV are distinct disease entities.

\section{Acknowledgements}

Not applicable.

\section{Funding}

This work was supported by the National Natural Science Foundation of China (grant nos. 81402453 and 81800641), the Natural Science Foundation of Hunan Province (grant no. 2015JJ4058), the Project of Hunan Health Commission of Hunan Province (grant no. C2019184) and the Chinese Society of Nephrology (grant no. 18020010780).

\section{Availability of data and materials}

The datasets used or analyzed during the present study are available from the corresponding author on reasonable request. 


\section{Authors' contributions}

YZ and PX designed, supervised the study, drafted and revised the manuscript. YZ and PX can authenticate the raw data in the present study. TW designed the research, collected, analyzed and interpreted the data, prepared figures and drafted the manuscript. JDO, CS and TM collected, analyzed and interpreted the data, edited and revised the manuscript. YOZ, JBC, ZX, XA, WL, XX, QZ collected and analyzed the data and revised the manuscript. All authors read and approved the final manuscript.

\section{Ethics approval and consent to participate}

The present study was approved by the ethics review committee of Xiangya Hospital Central South University (Changsha, China; approval no. 201403061).

\section{Patient consent for publication}

Not applicable.

\section{Competing interests}

The authors declare that they have no competing interests.

\section{References}

1. Falk RJ and Jennette JC: ANCA small-vessel vasculitis. J Am Soc Nephrol 8: 314-322, 1997.

2. Fries JF, Hunder GG, Bloch DA, Michel BA, Arend WP, Calabrese LH, Fauci AS, Leavitt RY, Lie JT, Lightfoot RW Jr, et al: The American college of rheumatology 1990 criteria for the classification of vasculitis. Summary. Arthritis Rheum 33: 1135-1136, 1990.

3. Jennette JC, Falk RJ, Bacon PA, Basu N, Cid MC, Ferrario F, Flores-Suarez LF, Gross WL, Guillevin L, Hagen EC, et al: 2012 revised international chapel hill consensus conference nomenclature of vasculitides. Arthritis Rheum 65: 1-11, 2013.

4. Lyons PA, Rayner TF, Trivedi S, Holle JU, Watts RA, Jayne DR, Baslund B, Brenchley P, Bruchfeld A, Chaudhry AN, et al: Genetically distinct subsets within ANCA-associated vasculitis. N Engl J Med 367: 214-223, 2012.

5. Hilhorst M, van Paassen P and Tervaert JW; Limburg Renal Registry: Proteinase 3-ANCA vasculitis versus myeloperoxidase-ANCA vasculitis. J Am Soc Nephrol 26: 2314-2327, 2015.

6. Fussner LA, Hummel AM, Schroeder DR, Silva F, Cartin-Ceba R, Snyder MR, Hoffman GS, Kallenberg CG, Langford CA, Merkel PA, et al: Factors determining the clinical utility of serial measurements of antineutrophil cytoplasmic antibodies targeting proteinase 3. Arthritis Rheumatol 68: 1700-1710, 2016.

7. Deshayes S, Martin Silva N, Khoy K, Yameogo S, Mariotte D, Lobbedez T and Aouba A: Clinical impact of subgrouping ANCA-associated vasculitis according to antibody specificity beyond the clinicopathological classification. Rheumatology (Oxford) 58: 1731-1739, 2019.

8. Hong Y, Shi P, Liu X, Yang L, Li K, Xu F, Liang S, Liu Z, Zhang $\mathrm{H}$, Chen $\mathrm{Y}$ and $\mathrm{Hu} \mathrm{W}$ : Distinction between MPO-ANCA and PR3-ANCA-associated glomerulonephritis in Chinese patients: A retrospective single-center study. Clin Rheumatol 38: 1665-1673, 2019.

9. Mukhtyar C, Lee R, Brown D, Carruthers D, Dasgupta B, Dubey S, Flossmann O, Hall C, Hollywood J, Jayne D, et al: Modification and validation of the birmingham vasculitis activity score (version 3). Ann Rheum Dis 68: 1827-1832, 2009.

10. Chen M, Xing GQ, Yu F, Liu G and Zhao MH: Complement deposition in renal histopathology of patients with ANCA-associated pauci-immune glomerulonephritis. Nephrol Dial Transplant 24: 1247-1252, 2009.
11. Berden AE, Ferrario F, Hagen EC, Jayne DR, Jennette JC, Joh K, Neumann I, Noël LH, Pusey CD, Waldherr R, et al: Histopathologic classification of ANCA-associated glomerulonephritis. J Am Soc Nephrol 21: 1628-1636, 2010.

12. Chen YX, Xu J, Pan XX, Shen PY, Li X, Ren H, Chen XN, Ni LY, Zhang W and Chen N: Histopathological classification and renal outcome in patients with antineutrophil cytoplasmic antibodies-associated renal vasculitis: A study of 186 patients and metaanalysis. J Rheumatol 44: 304-313, 2017.

13. Yates M, Watts RA, Bajema IM, Cid MC, Crestani B, Hauser T, Hellmich B, Holle JU, Laudien M, Little MA, et al: EULAR/ERA-EDTA recommendations for the management of ANCA-associated vasculitis. Ann Rheum Dis 75: 1583-1594, 2016.

14. Huang L, Zhong Y, Ooi JD, Zhou YO, Zuo X, Luo H, Chen JB, Wu T, Yang Y, Meng T, et al: The effect of pulse methylprednisolone induction therapy in Chinese patients with dialysis-dependent MPO-ANCA associated vasculitis. Int Immunopharmacol 76: $105883,2019$.

15. Watts RA, Mooney J, Skinner J, Scott DG and Macgregor AJ: The contrasting epidemiology of granulomatosis with polyangiitis (Wegener's) and microscopic polyangiitis. Rheumatology (Oxford) 51: 926-931, 2012.

16. Furuta S, Chaudhry AN, Hamano Y, Fujimoto S, Nagafuchi H, Makino H, Matsuo S, Ozaki S, Endo T, Muso E, et al: Comparison of phenotype and outcome in microscopic polyangiitis between Europe and Japan. J Rheumatol 41: 325-333, 2014.

17. Furuta $S$, Chaudhry AN, Arimura Y, Dobashi H, Fujimoto S, Homma S, Rasmussen N and Jayne DR: Comparison of the phenotype and outcome of granulomatosis with polyangiitis between UK and Japanese cohorts. J Rheumatol 44: 216-222, 2017.

18. Watts RA, Lane SE, Scott DG, Koldingsnes W, Nossent H, Gonzalez-Gay MA, Garcia-Porrua C and Bentham GA: Epidemiology of vasculitis in Europe. Ann Rheum Dis 60: 1156-1157, 2001.

19. Liu LJ, Chen M, Yu F, Zhao MH and Wang HY: Evaluation of a new algorithm in classification of systemic vasculitis. Rheumatology (Oxford) 47: 708-712, 2008.

20. Wu T, Peng J, Meng T, Liu Q, Ao X, Lin W, Yin H, Chen J, $\mathrm{Pu}$ J, Peng Z, et al: Clinicopathological features and prognostic analysis of 49 cases with crescentic glomerulonephritis. Exp Ther Med 18: 3984-3990, 2019.

21. Scott J, Hartnett J, Mockler D and Little MA: Environmental risk factors associated with ANCA associated vasculitis: A systematic mapping review. Autoimmun Rev 19: 102660, 2020.

22. Chang DY, Li ZY, Chen M and Zhao MH: Myeloperoxidase-ANCA-positive granulomatosis with polyangiitis is a distinct subset of ANCA-associated vasculitis: A retrospective analysis of 455 patients from a single center in China. Semin Arthritis Rheum 48: 701-706, 2019.

23. Pearce FA, Craven A, Merkel PA, Luqmani RA and Watts RA: Global ethnic and geographic differences in the clinical presentations of anti-neutrophil cytoplasm antibody-associated vasculitis. Rheumatology (Oxford) 56: 1962-1969, 2017.

24. Ponte C, Agueda AF and Luqmani RA: Clinical features and structured clinical evaluation of vasculitis. Best Pract Res Clin Rheumatol 32: 31-51, 2018.

25. Franssen C, Gans R, Kallenberg C, Hageluken C and Hoorntje S: Disease spectrum of patients with antineutrophil cytoplasmic autoantibodies of defined specificity: Distinct differences between patients with anti-proteinase 3 and anti-myeloperoxidase autoantibodies. J Intern Med 244: 209-216, 1998.

26. Falk RJ, Hogan S, Carey TS and Jennette JC: Clinical course of anti-neutrophil cytoplasmic autoantibody-associated glomerulonephritis and systemic vasculitis. The glomerular disease collaborative network. Ann Intern Med 113: 656-663, 1990.

27. Homma S, Suzuki A and Sato K: Pulmonary involvement in ANCA-associated vasculitis from the view of the pulmonologist. Clin Exp Nephrol 17: 667-671, 2013.

28. Franssen CF, Gans RO, Arends B, Hageluken C, ter Wee PM, Gerlag PG and Hoorntje SJ: Differences between anti-myeloperoxidase- and anti-proteinase 3-associated renal disease. Kidney Int 47: 193-199, 1995.

29. Kemna MJ, Damoiseaux J, Austen J, Winkens B, Peters J, van Paassen P and Cohen Tervaert JW: ANCA as a predictor of relapse: Useful in patients with renal involvement but not in patients with nonrenal disease. J Am Soc Nephrol 26: 537-542, 2015. 
30. Sinico RA, Radice A, Corace C, DI Toma L and Sabadini E: Value of a new automated fluorescence immunoassay (EliA) for PR3 and MPO-ANCA in monitoring disease activity in ANCA-associated systemic vasculitis. Ann N Y Acad Sci 1050: 185-192, 2005.

31. Liu Y, Zhang Y, Liu D, Tan X, Tang X, Zhang F, Xia M, Chen G, He L, Zhou L, et al: Prediction of ESRD in IgA nephropathy patients from an Asian cohort: A random forest model. Kidney Blood Press Res 43: 1852-1864, 2018.

32. Li D, Zou L, Feng Y, Xu G, Gong Y, Zhao G, Ouyang W, Thurman JM and Chao W: Complement factor B production in renal tubular cells and its role in sodium transporter expression during polymicrobial sepsis. Crit Care Med 44: e289-e299, 2016

33. Xiao H, Schreiber A, Heeringa P, Falk RJ and Jennette JC: Alternative complement pathway in the pathogenesis of disease mediated by anti-neutrophil cytoplasmic autoantibodies. Am J Pathol 170: 52-64, 2007.

34. Fukui S, Iwamoto N, Takatani A, Igawa T, Shimizu T, Umeda M, Nishino A, Koga T, Kawashiri SY, Tamai M, et al: Antineutrophilic cytoplasmic antibody-associated vasculitis with and without renal involvement: $\mathrm{C} 3$ contributes to prognosis, but renal involvement does not. Int J Rheum Dis 22: 789-796, 2019.

35. Choi H, Kim Y, Jung SM, Song JJ, Park YB and Lee SW: Low serum complement 3 level is associated with severe ANCA-associated vasculitis at diagnosis. Clin Exp Nephrol 23: 223-230, 2019.

36. Liu H, Peng Y, Liu H, Liu Y, Yuan S, Liu F, Yang D, Chen X, $\mathrm{He} \mathrm{L}, \mathrm{Fu}$ M, et al: Renal biopsy findings of patients presenting with isolated hematuria: Disease associations. Am J Nephrol 36: 377-385, 2012.

37. Hauer HA, Bajema IM, van Houwelingen HC, Ferrario F, Noël LH, Waldherr R, Jayne DR, Rasmussen N, Bruijn JA and Hagen EC; European Vasculitis Study Group (EUVAS): Renal histology in ANCA-associated vasculitis: Differences between diagnostic and serologic subgroups. Kidney Int 61: 80-89, 2002.

38. Cordova-Sanchez BM, Mejía-Vilet JM, Morales-Buenrostro LE, Loyola-Rodríguez G, Uribe-Uribe NO and Correa-Rotter R:
Clinical presentation and outcome prediction of clinical, serological, and histopathological classification schemes in ANCA-associated vasculitis with renal involvement. Clin Rheumatol 35: 1805-1816, 2016.

39. Rihova Z, Jancova E, Merta M, Rysava R, Reiterova J, Zabka J and Tesar V: Long-term outcome of patients with antineutrophil cytoplasmic autoantibody-associated vasculitis with renal involvement. Kidney Blood Press Res 28: 144-152, 2005.

40. Mohammad AJ and Segelmark M: A population-based study showing better renal prognosis for proteinase 3 antineutrophil cytoplasmic antibody (ANCA)-associated nephritis versus myeloperoxidase ANCA-associated nephritis. J Rheumatol 41: 1366-1373, 2014

41. de Joode AA, Sanders JS and Stegeman CA: Renal survival in proteinase 3 and myeloperoxidase ANCA-associated systemic vasculitis. Clin J Am Soc Nephrol 8: 1709-1717, 2013.

42. Ma Y, Han F, Chen L, Wang H, Han H, Yu B, Xu Y and Chen J: The impact of intravenous methylprednisolone pulses on renal survival in anti-neutrophil cytoplasmic antibody associated vasculitis with severe renal injury patients: A retrospective study. BMC Nephrol 18: 381, 2017.

43. Hauer HA, Bajema IM, Van Houwelingen HC, Ferrario F, Noël LH, Waldherr R, Jayne DR, Rasmussen N, Bruijn JA and Hagen EC; European Vasculitis Study Group (EUVAS): Determinants of outcome in ANCA-associated glomerulonephritis: A prospective clinico-histopathological analysis of 96 patients. Kidney Int 62: 1732-1742, 2002.

(i) $€$ This work is licensed under a Creative Commons Attribution-NonCommercial-NoDerivatives 4.0 International (CC BY-NC-ND 4.0) License. 